\title{
MORITA DUALITY FOR GROTHENDIECK CATEGORIES
}

\author{
J. L. Gómez Pardo and P. A. Guil Asensio \\ Dedicated to the memory of Professor Pere Menal
}

\begin{abstract}
We survey some recent results on the theory of Morita duality for Grothendieck categories, comparing two different versions of this concept, and giving applications to $Q F-3$ and $Q f-3$ ' rings.
\end{abstract}

In recent years, two different extensions of the concept of Morita duality to the framework of Grothendieck categories have been proposed. These extensions have been motivated, on the one hand, by the desire of providing a unified background to now Morita-like dualities that have appeared in the literature (see e.g. [2], [15], [21]) and, on the other, by the realization that Morita duality for Grothendieck categories can, in fact, be applied to the study of rings and modules when one considers, say, a module category and looks at Morita dualities of some convenient quotient categorics. This is the underlying idca in a series of papers by Colby and Fuller [4], [5], [6] in which they apply these techniques to the study of $Q F-3$ and $Q F-3^{\prime}$ rings. In this paper, we survey some recent results on this topic, that have been obtained in [9] and [10].

The first definition of Morita dualities for Grothendieck categories is due to Colby and Fuller [4], so we are going to speak of Colby-Fuller dualities. A right adjoint pair of contravariant functors $D: \mathcal{A} \rightleftarrows \mathcal{A}^{\prime}: D^{\prime}$ between abelian categories $\mathcal{A}$ and $\mathcal{A}^{\prime}$ defines a Colby-Fuller duality if both functors are exact and the full subcategories $\mathcal{A}_{0}$ and $\mathcal{A}_{0}^{\prime}$ of reflexive objects of $\mathcal{A}$ and $\mathcal{A}^{\prime}$, are closed under subobjects, quotient objects and finite direct sums (i.e., thcy are finitely closed) and contain generating sets for $\mathcal{A}$ and $\mathcal{A}^{\prime}$, respectively (i.e, they are generating). The second extension has been recently proposed by Anh and Wiegandt in [3]. There

Work partially supported by the DGICYT (PB90-0300). 
is an Anh-Wiegandt duality between two Grothendieck categories $\mathcal{A}$ and $\mathcal{A}^{\prime}$ if there exist finitely closed generating full subcategories $\varphi$ and $\varphi^{\prime}$ of $\mathcal{A}$ and $\mathcal{A}^{\prime}$, respectively, and a contravariant equivalence $F: \varphi \rightleftarrows \varphi^{\prime}: F^{\prime}$. In this case, the objects of $\varphi$ and $\varphi^{\prime}$ are also called reflexive.

It is easy to check that any Colby-Fuller duality between Grothendieck categories defines an Anh-Wiegandt duality, but the converse is not so clear, even when it is known to be true for module categorics. In fact, several natural questions arise at this point:

Question 1. Do Colby-Fuller and Anh-Wirgandt dualities coincide?.

We will see below that the answer is no in general, but that they coincide in several important cases. This question is very close to a harder problem posed by Colby and Fuller in [4] and [6]:

Question 2. Is the exactness condition for the right adjoint functors of a Colby-Fuller duality a consequence of the remaining hypotheses?.

We will also give a. negative answer to this problem. Other natural questions are the following:

Question 3. Can the usual characterizations of Morita duality for module categories in terms of linear compactness be extended to these dualities?.

Question 4. What happens when one of the Grothendieck categories is a module category?.

We will show that Question 3 has a satisfactory answer and, regarding Question 4, that if a Grothendieck category has a Colby-Fuller duality with a module category, then it is a module category too; whilst for Anh-Wiegandt dualities we exhibit a counterexample to this situation. Finally, we give some examples of the applications of this theory to the study of $Q F-3$ and $Q F-3$ ' rings.

\section{Preliminaries and notation}

Throughout, $R$ denotes an associative unitary ring and $R$-Mod (Mod$R$ ), the category of left (resp. right) $R$-modulcs. A ring $R$ is said to be lcft $Q F-3$ if it has a minimal faithful left $R$-modulc (i.c., a faithful module which is a direct summand of any other faithful module) and it is left $Q F-3$ ' when its left injective hull, $E\left({ }_{R} R\right)$, is torsionless. In case that $R$ is left and right $Q F-3\left(Q F-3^{\prime}\right)$, we will simply say that it is $Q F-3$ (resp. $Q F-3^{\prime}$ ) and we will use a similar convention for other classes of rings.

Recall that a pair of contravariant functors $D: \mathcal{A} \rightleftarrows \mathcal{A}^{\prime}: D^{\prime}$ between abelian categories $\mathcal{A}$ and $\mathcal{A}^{\prime}$ is called right adjoint if there are natural isomorphisms $\eta_{A, A^{\prime}}: \operatorname{Hom}_{\mathcal{A}}\left(A, D^{\prime} A^{\prime}\right) \rightarrow \operatorname{Hom}_{\mathcal{A}^{\prime}}\left(A^{\prime}, D A\right)$, for each pair 
of objects $A \in A$ and $A^{\prime} \in \mathcal{A}^{\prime}$. Associated to $\eta$ there are the arrows of right adjunction, $\tau: 1_{\mathcal{A}} \rightarrow D^{\prime} D$ and $\tau^{\prime}: 1_{\mathcal{A}^{\prime}} \rightarrow D \circ D^{\prime}$, defined as $\tau_{A}=\eta_{A, D A}^{-1}\left(1_{A}\right)$ and $\tau_{A^{\prime}}^{\prime}=\eta_{D^{\prime} A^{\prime}, A^{\prime}}\left(1_{D^{\prime} A^{\prime}}\right)$ (see [4]). An object $A$ of $\mathcal{A}$ ( $A^{\prime}$ of $\mathcal{A}^{\prime}$ ) is said to be reflexive if $\tau_{A}$ (resp. $\tau_{A^{\prime}}$ ) is an isomorphism.

We will denote by $\mathcal{D}\left(\mathcal{D}^{\prime}\right)$ the Lambek localizing subcategory of $R$-Mod (Mod- $R$ ) associated to the filter of dense left (resp. right) ideals. We will follow, as in [4], the convention for the endomorphism ring of an object $A$ in an abelian category $\mathcal{A}$ of calling $\operatorname{End}_{\mathcal{A}}(A)=\operatorname{Hom}_{\mathcal{A}}(A, A)^{\text {op }}$. We refer to $[1]$ and $[20]$ for any undefined concept used in this paper.

\section{Examples}

We begin by giving a result that highlights the close relationship that exists between Question 1 and Question 2 .

Theorem 2.1. Let $F: \varphi \rightleftarrows \varphi^{\prime}: F^{\prime}$ be an Ahn-Wiegandt duality between Grothendieck categories $\mathcal{A}$ and $\mathcal{A}^{\prime}$. Then $F$ and $F^{\prime}$ can be extended to a right adjoint pair of contravoriant functors $D: \mathcal{A} \rightleftarrows \mathcal{A}^{\prime}: D^{\prime}$ in a unique way.

Sketch of proof: For the extension, observe that any object $A$ of $\mathcal{A}$ can be written as $A=\underline{\lim } A_{i}$, where the $A_{i}$ are reflexive subobjects of $A$. Dcfine then $D A=\overleftrightarrow{\lim } F A_{2}$ and similarly $D^{\prime}$. One may then check that this definition is independent of the representation of $A$ as direct limit of objects of $\varphi$ and that, in fact, $D$ and $D^{\prime}$ are a right adjoint pair of contravariant functors.

It might appear, at first sight, that Theorem 2.1 makes Question 1 and Question 2 equivalent but this is not the case. The reason is that, when one extcnds an Anh-Wiegandt duality to a contravariant right adjoint pair, the enlarged subcatcgories of reflexive objects $\mathcal{A}_{0}$ and $\mathcal{A}_{0}^{\prime}$ (which contain $\varphi$ and $\varphi^{\prime}$, respectively) are not necessarily finitely closed. This is shown by the following simple example that proves that Colby-Fuller dualities are stronger than Anh-Wiegandt dualitics.

Example 2.2. Let $\mathcal{A}$ be the Grothendieck category of all abelian $p$-groups and let $D: \mathcal{A} \rightarrow \mathcal{A}$-be the (composition) functor $D=$ $T\left(\operatorname{Hom}_{\mathbb{Z}}\left(-, \mathbb{Z}\left(p^{\infty}\right)\right)\right)$, where $T$ denotes the torsion functor of abelian groups and $\mathbb{Z}\left(p^{\infty}\right)$ is the Prufer group. Then, $D$ forms a right adjoint pair with itself, and the full subcatcgory $\varphi$ of $\mathcal{A}$ consisting of the artinian $p$-groups is finitely closed and generating. Further, $D$ establishes a contravariant equivalence of $\varphi$ with itsclf and, thus, it defines an Anh-Wiegandt duality in $\mathcal{A}$. However, the functor $D$ is not exact 
and so, it does not define a Colby-Fuller duality. In fact, one may check that, if $i: \mathbb{Z}\left(p^{n}\right) \rightarrow \mathbb{Z}\left(p^{\infty}\right)$ is the inclusion of the cyclic $p$-group $\mathbb{Z}\left(p^{n}\right)$ in $\mathbb{Z}\left(p^{\infty}\right), D(i)$ is not an epimorphism, since $D\left(\mathbb{Z}\left(p^{n}\right)\right) \cong \mathbb{Z}\left(p^{n}\right)$ and $D\left(\mathbb{Z}\left(p^{\infty}\right)\right)=0$. But this adjoint pair has also another pathology that prevents it from being a Colby-Fuller duality. Indeed, $T\left(\prod_{n=1}^{\infty} \mathbb{Z}\left(p^{n}\right)\right)$ is a reflexive object in $\mathcal{A}$ (i.e., it belongs to $\mathcal{A}_{0}$ ) but has a subobject, $\oplus_{n=1}^{\infty} \mathbb{Z}\left(p^{n}\right)$, which is not refiexive. Thus, the full subcategories of reflexive objects are not finitely closed.

Example 2.2 provides a negative answer to Question 1 but, since $\mathcal{A}_{0}$ is not finitely closed, it says nothing about Question 2 , i.e., it does not solve the problem posed by Colby-Fuller in [4, pp. 551-552] and [6, p. 185] asking whether the exactness of the functors is a superfluous hypothesis in the definition of a Colby-Fuller duality (recall that this is just the case for Morita dualities between module categories). The following example completely solves this problem.

Example 2.3. Let, as in Example 2.2, $\mathcal{A}$ be the Grothendieck category of all abelian $p$-groups and let $\mathbb{Z}_{p}$ denote the ring of $p$-adic integers. Consider the contravariant functors

$$
D=\operatorname{Hom}_{\mathbb{Z}}\left(-, \mathbb{Z}\left(p^{\infty}\right)\right): \mathcal{A} \rightleftarrows \mathbb{Z}_{p}-\operatorname{Mod}: T\left(\operatorname{Hom}_{\mathbb{Z}_{p}}\left(-, \mathbb{Z}\left(p^{\infty}\right)\right)=D^{\prime}\right.
$$

Then, the functors $D$ and $D^{\prime}$ are right adjoint and it is not diffcult to see that they induce a contravariant equivalence between the full subcategories of $\mathcal{A}$ and $\mathbb{Z}_{p}$-Mod consisting in the artinian $p$-groups and the noetherian $p$-adic modules, respectively. Further, by an argument similar to Example 2.2, $D^{\prime}$ is not exact. Now, we only need to show that any reflexive object of $\mathbb{Z}_{p}$-Mod is noetherian. But, by the structure theorem of Fuchs for character groups (see [7, Theorem 47.1]), if $X$ is a reflexive object of $\mathbb{Z}_{p}$-Mod, $X \cong \prod_{I} \mathbb{Z}\left(p^{n_{i}}\right) \ominus \prod_{J} \mathbb{Z}_{p}$ and hence it suffices to prove that $I$ and $J$ must be finite. If this is not the case, let $\kappa=\max (|I|,|J|) \geq \aleph_{0}$. Then $\left.\operatorname{Hom}_{\mathbb{Z}_{p}}\left(\left(\underset{I}{\mathbb{Z}}\left(p^{n_{i}}\right)\right) \oplus \underset{J}{(\oplus \mathbb{Z}} \mathbb{Z}_{p}\right), \mathbb{Z}\left(p^{\infty}\right)\right)$ contains a subgroup $A \cong \prod_{n} \mathbb{Z}(p)$, so that $|A|=2^{\kappa}$ and $p A=0$. By the injectivity of the p-adic module $\mathbb{Z}\left(p^{\infty}\right)$ one can then show that $D^{\prime} X$ contains a subgroup of cardinality $\geq 2^{\kappa}$ and hence $\left|D D^{\prime}(X)\right| \geq 2^{2^{\kappa}}$. Since $|X|=2^{\kappa}$, this shows that $X$ cannot be reficxive and hence $I$ and $J$ must be both finite.

Remark. Observe that $\mathbb{Z}_{p}$-Mod has a self Morita duality. Since $\mathcal{A}$ cannot be equivalent to $\mathbb{Z}_{p}$-Mod, Example 2.3 also shows that a. Grothendieck category (or even a module category with Morita dual- 
ity) may have Anh-Wiegandt dualities with nonequivalent Grothendieck categories.

\section{Linear compactness for Grothendieck categories}

In Examples 2.2 and 2.3, we have shown that Anh-Wiegandt dualities are weaker than Colby-Fuller dualities. However, it is well known that they coincide for module categories. In this section we are going to obtain conditions under which an Anh-Wiegandt duality can be extended to a Colby-Fuller duality. We recall from $[\mathbf{8}]$ that an object $A$ of a Grothendieck category $\mathcal{A}$ is called linearly compact when, for each inverse system of epimorphisms $\left\{p_{i}: X \rightarrow X_{i}\right\}_{I}$ in $A$, the induced morphism $\lim p_{i}: X \rightarrow \lim X_{8}$ is also an epimorphism. We will say that a. subcategory $\mathcal{C}$ of $\overleftarrow{A}$ is linearly compact if, for each epimorphism of inverse systems $\left\{p_{i}: X_{i} \rightarrow Y_{i}\right\}_{I}$ in $\mathcal{A}$, with the $X_{i}$ in $\mathcal{C}$, the induced morphism $\lim _{\llcorner} p_{i}$ is an epimorphism. We remark that any object of a linearly compact subcategory of $\mathcal{A}$ is linearly compact but, as we will sce below, the converse is not true. We begin with the following proposition that is easy to prove (sec also [10, Lemma 2]):

Proposition 3.1. Let $D: \mathcal{A} \rightleftarrows \mathcal{A}^{\prime}: D^{\prime}$ be a right adjoint pair of contravariant functors between Grothendieck categories $\mathcal{A}$ and $\mathcal{A}^{\prime}$ and let $\varphi$ and $\varphi^{\prime}$ be finitely closed full subcategories of $\mathcal{A}$ and $\mathcal{A}^{\prime}$ consisting of reflexive objects. Then any object of $\varphi$ or $\varphi^{\prime}$ is linearly compact.

Now, we are in position to prove the following theorem:

Theorem 3.2. Let $D: \mathcal{A} \rightleftarrows \mathcal{A}^{\prime}: D^{\prime}$ be a right adjoint pair of contravariant functors between Grothendieck categories. The following conditions are equivalent:

i) There exist finitely closed, generating, linearly compact full subcategories $\varphi$ and $\varphi^{\prime}$ of $\mathcal{A}$ and $\mathcal{A}^{\prime}$ such that $D$ and $D^{\prime}$ induce a contravariant equivalence between them.

ii) $D$ and $D^{\prime}$ define a Colby-Fuller duality between $\mathcal{A}$ and $\mathcal{A}^{\prime}$.

Further, when these equivalent conditions hold, the reflexive objects for the right adjunction coincide with the linearly compact objects.

Sketch of proof: i) $\Rightarrow$ ii) First, notice that since we already know that $D$ and $D^{\prime}$ arc left exact (by the adjunction), in order to show that they are exact we only need to prove that they transform monomorphisms in epimorphisms. But this is a consequence of the fact that $\varphi$ and $\varphi^{\prime}$ are linearly compact and generating and $D$ and $D^{\prime}$ establish a contravariant 
equivalence between them. Now, using an argument similar to [4, Prop. 2.3 i)], we can check that $D$ and $D^{\prime}$ are faithful and, applying [6, Prop. 4], we see that $D$ and $D^{\prime}$ define a Colby-Fuller duality.

ii) $\Rightarrow$ i) We only have to show that the full subcategories $\mathcal{A}_{0}$ and $\mathcal{A}_{0}^{\prime}$ of $\mathcal{A}$ and $\mathcal{A}^{\prime}$ consisting in the reflexive objects for the adjunction are linearly compact. Consider an epimorphism of inverse systems $\left\{p_{i}: X_{i} \rightarrow Y_{i}\right\}_{I}$ in $\mathcal{A}$ with each $X_{i}$ in $\mathcal{A}_{0}$. Then, as $\left\{D p_{i}\right\}$ is a monomorphism of direct systems in $\mathcal{A}^{\prime}$, we get that $\lim D p_{i}$ is a monomorphism. Now, since $D^{\prime}$ is exact, we see that $\lim _{\longleftarrow} D^{\prime} \overrightarrow{D p_{i}} \cong D^{\prime}\left(\lim D p_{i}\right)$ is an epimorphism. But, as all the $X_{\imath}$ are reficxive, it is easy to see that $D^{\prime} D p_{i} \cong p_{i}$ canonically. Similarly, $\mathcal{A}_{0}^{\prime}$ is linearly compact.

Finally, observe that, since $\mathcal{A}_{0}$ and $\mathcal{A}_{0}^{\prime}$ are finitely closed, any reflexive object is linearly compact by Proposition 3.1. To prove the converse it is enough to write the dual of any linearly compact object as a direct limit of reflexive subobjects and then use the linear compactness and the exactness of $D$ and $D^{\prime}$.

Remark. In view of Theorem 3.2 it is clear that the category of artinian p-groups considered in Examples 2.3 and 2.4 is not a linearly compact subcategory of the category of abelian $p$-groups, despite the fact that each artinian p-group is linearly compact.

If we combine Theorem 2.1 and Theorem 3.2 , we get a necessary and sufficient condition for both kind of dualities to agree.

Corollary 3.3. An Anh-Wiegandt duality $F: \varphi \rightleftarrows \varphi^{\prime}: F^{\prime}$ between Grothendieck categories $\mathcal{A}$ and $\mathcal{A}^{\prime}$ can be extended to a Colby-Fuller duality if, and only if, $\varphi$ and $\varphi^{\prime}$ are linearly compact subcategories of $\mathcal{A}$ and $\mathcal{A}^{\prime}$, respectively.

The reason for the fact that for modulc categories both kinds of dualities coincide is that a full subcategory of a category of modules is linearly compact if and only if all his objects are linearly compact (see e.g. [11, Theorem 7.1] and [18, Lemma 5.7]). The following corollary extends this fact.

Corollary 3.4. Let $F: \varphi \rightleftarrows \varphi^{\prime}: F^{\prime}$ be an Anh-Wiegandt duality between Grothendieck categories $\mathcal{A}$ and $\mathcal{A}^{\prime}$ which contain generating sets of projective objects. Then this duality can be extended to a (unique) Colby-Fuller duality between $\mathcal{A}$ and $\mathcal{A}^{\prime}$.

Sketch of proof: By the Gabricl-Popescu Theorem [20, Theorem X.4.1] the categories $\mathcal{A}$ and $\mathcal{A}^{\prime}$ can be represented as quotient categoxies of module categories and, since they contain generating sets of projective 
objects, the functors of the representation can be chosen to be exact. Now the linear compactness of $\varphi$ and $\varphi^{\prime}$ is a consequence of Proposition 3.1 and the fact that, for module categories, any full subcategory consisting of linearly compact objects is linearly compact.

Remark. We note that this is just the situation considered in [2], [15] and [21].

We finalize this section with a theorem that extends Muller's characterization of Morita dualities [17, Theorem 1] to Colby-Fuller dualities with reflexive generators. Observe that this particular case of ColbyFuller dualities is of special interest because of its application to the study of $Q F-3$ and $Q F-3$ ' rings (see [4], [5] and [6]). First, we need the following result that is implicit in [6] (see also [4]):

Theorem 3.5 (Colby-Fuller). A ring $R$ is $Q F^{\prime}-3$ ' iff the $R$-dual functors induce a Colby-Fuller duality with reflexive generators between $R-\operatorname{Mod} / \mathcal{D}$ and $\operatorname{Mod}-R / \mathcal{D}^{\prime}$. Further, $R$ is also its own maximal quotient ring if only if it is closed for the localizing subcategories $\mathcal{D}$ and $\mathcal{D}^{\prime}$.

Theorem 3.6. Let $\mathcal{A}$ be a Grothendieck category. The following are equivalent:

i) There exists a Grothendieck category $\mathcal{A}^{\prime}$ and a Colby-Fuller duality $D: \mathcal{A} \rightleftarrows \mathcal{A}^{\prime}: D^{\prime}$ with reflexive generators.

ii) A has a generator-cogenerator $U$ such that the smallest finitely closed subcategory of $\mathcal{A}$ containing $U$ is linearly compact.

Skecth of proof: i) $\Rightarrow$ ii) By Proposition 3.1 and [4, Proposition 2.3]. ii) $\Rightarrow$ i) Take $R=\operatorname{End}_{\mathcal{A}}(U)$. By [16, Theorem 8.3$], R$-Mod $/ \mathcal{D}$ is equivalent to $\mathcal{A}$, where $D$ is the Lambek localizing subcategory of $R$-Mod and $R$ is left $Q F-3$ ' and its own left maximal quotient ring. Further, identifying $R$-Mod $/ \mathcal{D}$ with $\mathcal{A}$, we get that the smallest finitely closed subcategory of $R$-Mod $/ \mathcal{D}$ containing $R$ is linearly compact. We want to see that the $R$-double dual functor ()$^{* *}: \operatorname{Mod}-R \rightarrow \operatorname{Mod}-R$ preserves monomorphisms or, equivalently, that if $j$ is a monomorphism in Mod- $R$, then $j^{*}$ is an epimorphism in $R$-Mod $/ \mathcal{D}$. We are going to prove it in five steps:

Step 1. Consider the inclusion $j: J \rightarrow R_{R}$ where $J=\sum_{i=1}^{n} a_{i} R$ is a finitely generated right idcal of $R$. If $j^{* *}$ is not a monomorphism, there is a non zero homomorphism $\varphi: J^{*} \rightarrow{ }_{R} R$ such that $\varphi \circ j^{*}=$ 0 . Let $f \in J^{*}$ such that $\varphi(f) \neq 0$ and define $\psi: J^{*} \rightarrow{ }_{R} R^{n}$ the monomorphism given by $\psi(f)=\left(f\left(a_{i}\right)\right)_{i=1 \ldots n}$. Then, since the injective envelope of $R_{R} R, E\left({ }_{R} R\right)$, is torsionless, one can construct a homo- 
morphism $\alpha:{ }_{R} R^{n} \rightarrow{ }_{R} R$ such that $\alpha \circ \psi \neq 0$, but $\alpha \circ \psi \circ j^{*}=0$. Take $h \in J^{*}$ such that $\alpha \circ \psi(h) \neq 0$ and define $\bar{h}: \underset{i=1}{\oplus} a_{i} R \rightarrow R^{n}$ as $\bar{h}\left(r_{1}, \ldots, r_{n}\right)=\left(h\left(a_{1}\right) r_{\lambda}, \ldots, h\left(a_{n}\right) r_{n}\right)$. Then it is easy to see that $(\alpha \circ \psi)(h)=\alpha\left(h\left(a_{1}\right), \ldots, h\left(a_{n}\right)\right)=\alpha\left(\bar{h}\left(a_{1}, \ldots, a_{n}\right)\right)=h\left(\alpha\left(a_{1}, \ldots, a_{n}\right)=\right.$ $h\left(\alpha \circ \psi \circ j^{*}(1)\right)=0$, which is a contradiction. Thus, $j^{* *}$ is a monomorphism.

Step 2. If $J$ is a right ideal of $R$ and $j: J \rightarrow R$ is the inclusion, then wo can write $J: \lim _{\longrightarrow} J_{i}$ where the $J_{i}$ are finitely generated ideals of $J$. Now, using Step 1 and the fact that $R$ is linearly compact in $R$-Mod $/ \mathcal{D}$, it is easy to show that $j^{*}$ is also an epimorphism in $R$-Mod $/ \mathcal{D}$, i.e., $j^{* *}$ is a monomorphism.

Step 3. If $j: X \rightarrow R^{n}$ is a monomorphism in Mod- $R$, we can prove using induction and Step 2 that $j^{* *}$ is a monomorphism.

Step 4. If $j: X_{R} \rightarrow R_{R}^{(I)}$ is a monomorphism, for some set $I$, then we can write $j=\lim _{\longrightarrow} j_{F}: X_{F} \rightarrow R^{F}$, where $F$ ranges over the finite subsets of $I$, and use Step 3 and the linear compactness of the smallest finitely closed subcategory of $R$-Mod $/ \mathcal{D}$ containing $R$ to show that $j^{* *}$ is a monomorphism.

Step 5. Finally, if $j: X \rightarrow Y$ is an arbitrary monomorphism in Mod$R$, we can see that $j^{* *}$ is a monomorphism writing $Y$ as a quotient of $R^{(I)}$, considering the inverse image of $X$ by this epimorphism, and then using Step 4.

Thus, we have shown that ( $)^{* *}: \operatorname{Mod}-R \rightarrow$ Mod- $R$ preserves monomorphisms. Using now Theorem 3.5 and [6, Theorem 1], we sec that there is a Colby-Fuller duality between $R-\operatorname{Mod} / \mathcal{D}$ and $\operatorname{Mod}-R / \mathcal{D}^{\prime}$ with reflexive generators.

Combining Theorems 3.5 and 3.6, we get the following corollary that extends to $Q F-3^{\prime}$ maximal quotient rings the characterization of $Q F-3$ maximal quotient, rings given in [19. Theorem 2.1].

Corollary 3.7. A ring $R$ is $Q F-3^{\prime}$ and its own maximal quotient ring if and only if it is the endomorphism ring of a generator-cogenerator $U$ of a Grothendieck category such that the smallest finitely closed full subcategory containing $U$ is linearly compact.

\section{Applications to $Q F-3$ and $Q F-3$ ' rings}

In this section, we exhibit some consequences of the previous results for the study of $Q F-3$ and $Q F-3$ ' rings. We begin by answering the fourth question posed in the introduction. First, we need the following result, due to Colby and Fuller (see [5, Theorem 2]): 
Theorem 4.1 (Colby-Fuller). A ring $R$ is $Q F-3$ iff both $R$-dual functors preserve monomorphisms and the quotient categories $R$-Mod $/ \mathcal{D}$ and $\operatorname{Mod}-R / \mathcal{D}^{\prime}$ are (equivalent to) module categories.

Theorem 4.2. Let $D: \mathcal{A} \rightleftarrows \mathcal{A}^{\prime}: D^{\prime}$ be a Colby-Fuller duality between Grothendieck categories $\mathcal{A}$ and $\mathcal{A}^{\prime}$. If $\mathcal{A}$ is equivalent to a category of modules, then so is $\mathcal{A}^{\prime}$.

Sketch of proof: We know that $\mathcal{A}$ is equivalent to a category of modules, say $R$-Mod. Then, since $R$ is finitely generated, $R$ is a reflexive generator and, by Proposition 3.1, is linearly compact. Thus, ${ }_{R} R$ is semiperfect and there are only a finite number of isomorphism classes of injective hults of simple modules. Now, using an argument similar to [4, Prop. $2.3 \mathrm{i})]$ and the fact that the injective hulls of simple modules are finitely cogenerated, we get that $R$-Mod has a linearly compact cogenerator and, by $[17$, Theorem 1], it has a Morita duality. Finally, using Theorem 4.1 and [19, Theorem 2.1], it is easy to check that $\mathcal{A}^{\prime}$ is equivalent to a module category.

Remark. As pointed out in the remark following Example 2.3, the corresponding result for Anh-Wiegandt dualities is not true.

Now, we are ready to obtain some applications of the preceding results. We begin with the following theorem that is a consequence of Theorem 4.2, [6, Theorem 2] and the Morita-Ringel-Tachikawa Theorem $[19$, Theorem 2.1].

Theorem 4.3. R-Mod has a Morita duality if and only if it has a generator-cogenerator whose endomorphism ring is right $Q F-3$ :

Theorems 4.2 and 4.3 allow us to obtain new one-sided conditions for a (left and right) $Q F-3$ ' to be $Q F-3$. We recall that a faithful left $R$ module $P$ is called dominant when it is finitely generated projective and $P_{S}$ cogenerates all the simple modules, where $S=\operatorname{End}\left({ }_{R} P\right)$ (see [12]). In [13], it is shown that a $Q F-3$ ' ring has a left dominant module if and only if $R-\operatorname{Mod} / \mathcal{D}$ is equivalent to a category of modules. Combining these facts with Poposition 4.2 , we get

Corollary 4.4. The following conditions are equivalent:

i) $R$ is a $Q F-3$ ring.

ii) $R$ is $Q F-3^{\prime}$ and has a left (or right) dominant module.

Remark. Corollary 4.4 cannot be extended to one-sided $Q F-3$ ' rings: 
the ring $R=\left(\begin{array}{ll}\mathbb{Z} & \mathbb{Q} \\ 0 & \mathbb{Q}\end{array}\right)$ is a left $Q F-3$ ' ring with a left dominant module ${ }_{R} P=\left(\begin{array}{ll}0 & Q \\ 0 & Q\end{array}\right)$. However, it is not right $Q F-3$ ' nor left $Q F-3$.

Theorem 4.2 and 4.3 also can be used for obtaining new characterizations of particular classes of $Q F-3$ rings. We finalize this paper with two examples of them (see also [9]).

Corollary 4.5. The following conditions are equivalent:

i) $R$ is a $Q F-3$ ring with ascending chain condition in annihilators.

ii) $R$ is a $Q F-3^{\prime}$ ring with left (or right) perfect maximal quotient ring.

Corollary 4.6. The following conditions are equivalent:

i) $R$ is a nonsingular $Q F-3$ ring.

ii) $R$ is a $Q F-3^{\prime}$ ring with a faithful non-singular left module which has finite Goldie dimension.

Acknowledgement. The second author would like to acknowledge the hospitality of the Departament of Algebra of the University of Santiago.

\section{References}

1. F. W. ANDERSON AND K. R. FUller, "Rings and categoties of modules," Springer-Verlag, New York, 1974.

2. P. N. Ani AND C. Menini, Morita duality for rings with local units, Preprint.

3. P. N. ANH AND R. WIEGANDT, Morita duality for categories, Preprint.

4. R. R. Colby AND K. R. Fuller, Exactness of the double dual and Morita duality for Grothendieck categories, J. Algebra 82 (1983), $546-558$.

5. R. R. COLBY AND K. R. Fuller, On rings whose double dual functors preserve monomorphisms, J. Pure Appl. Algebra 28 (1983), 223-234.

6. R. R. COLBY AND K. R. FULLER, QF-3' rings and Morita duality, Tsukuba J. Math. 8 (1984), 183-188.

7. L. FucHS, "Infinite abelian groups," Vol. 1, Academic Press, New York, 1970. 
8. J. L. Gómez Pardo, Counterinjective modules and duality, $J$. Pure Appl. Algebra 61 (1989), 165-179.

9. J. L. Gómez Pardo nND P. A. Guil Asensio, $Q F-3$ rings and Morita duality, Comm. Algebra 18(8) (1990), 2755-2764.

10. J. L. Gómez PARdo and P. A. Guil Asensio, Linear compactness and Morita duality for Grothendieck categories, J. Algebra 148 (1992), 53-67.

11. C. U. JENSEN, "Les functeurs derivés de lim et leurs applications en théorie des modules," Lecture Notes in Math. 254, Springer-Verlag, Berlin, 1972.

12. T. Kato, Dominant modules, J. Algebra 14 (1970), 341-349.

13. T. KATO, Rings having dominant modules, Tôhoku Math. J. 24 (1972), 1-10.

14. E. MatLIs, Injective modules over Noetherian rings, Pacific $J$. Math. 8 (1958), 511-528.

15. C. MENini AND A. Del Río, Morita duality and graded rings, Comm. Algebra 19(6) (1991), 1765-1794.

16. K. Morita, Localization in categories of modules, I. Math. Z. 144 (1970), 121-144.

17. B. J. MüLlef, Lincar compactness and Morita duality, J. Algebra $16(1970), 60-66$.

18. U. OBERST, Duality theory for Grothendieck categories and linearly compact rings, J. Algebra 15 (1970), 473-542.

19. C. M. Ringel And H. TaChikawa, $Q F-3$ rings, J. Reine Angew Math. 272 (1975), 49-72.

20. B. St'Enström, "Rings of quotients," Springer-Verlag, Berlin, 1975.

21. K. Yamagata, On Morita duality for additive group-valued functors, Comm. Algebra 7(4) (1979), 367-392.

J. L. Gómez Pardo:

Departamento de Álxebra

Universidade de Santiago de Compostela

15771 Santiago de Compostela

SPAIN
P. A. Guil Asensio:

Departamento de Matemáticas

Universidad de Murcia

30100 Espinardo (Murcia)

SPAIN 